\title{
Measurements of photon and jet production properties with CMS
}

\author{
Giannis Flouris*† \\ University of Ioannina $(G R)$ \\ E-mail: giannis.flourisecern.ch
}

The measurements of the jet charge, the $\mathrm{Z}+$ jet and $\gamma+$ jet, the dijet azimuthal decorrelations, and the inclusive jet cross sections are presented. The measurements use the samples of proton-proton collisions collected with the CMS detector at the LHC at a center-of-mass energy of $\sqrt{s}=8$ and $\sqrt{s}=13 \mathrm{TeV}$.

Fourth Annual Large Hadron Collider Physics

13-18 June 2016

Lund, Sweden

* Speaker.

${ }^{\dagger}$ On behalf of the CMS Collaboration. 


\section{Introduction}

Jets are the long distance objects that are probes of quarks and gluons, as they are described in the framework of perturbative quantum chromodynamics (pQCD). Jets are recorded in the detectors as clustered streams of particles. pQCD becomes a powerful tool when utilizing the factorization theorem, but it introduces some free parameters that can only be constrained by experiment such as the coupling constant and the parton density functions (PDF). Furthermore, pQCD is only valid as long as the asymptotic freedom assumption is valid.

LHC is a factory of jets that allows to study the validity of pQCD and constrain the free parameters. Jets, in the LHC, are produced in a phase space that has never been explored before thus the test whether the asymptotic freedom assumption is valid is one of the most significant tests.

Five CMS [1] measurements are presented in this proceeding. The measurement of the jet charge in dijet events [2], the comparison of $\mathrm{Z} / \gamma^{*}+$ jets to $\gamma+$ jets cross section [3], the inclusive jet differential cross section [4], and the dijet azimuthal decorrelation [5] at $\sqrt{s}=8 \mathrm{TeV}$. Finally the first measurement of the inclusive jet differential cross section at $\sqrt{s}=13 \mathrm{TeV}[6]$ is presented.

\section{The Measurements}

CMS measured the jet charge observables in dijet events at $8 \mathrm{TeV}$ [2]. The measurement is performed differentially in the transverse momentum of the leading jet, $p_{\mathrm{T}}$, and compared with predictions from Monte Carlo (MC) generators. The analysis used the jets where the leading and subleading jets lie within $|\eta|<1.4$, with $p_{\mathrm{T}, 1}>400 \mathrm{GeV}$ and $p_{\mathrm{T}, 2}>100 \mathrm{GeV}$, where $p_{\mathrm{T}, \mathrm{i}}$ is the transverse momentum of leading $(i=1)$ and subleading $(i=2)$ jet. The jet charge is defined as:

$$
Q^{\kappa}=\frac{1}{\left(p_{T}\right)^{\kappa}} \sum_{i} Q_{i}\left(p_{T}^{i}\right)^{\kappa}
$$

which is the sum over all charged particles within the jet with $p_{T}>1 \mathrm{GeV}$ and charge $Q_{\mathrm{i}} ; \kappa$ is a free parameter.

The left part of Fig. 1 shows the distribution of the leading jet charge compared to distributions from simulated events where the jet initiated by an up quark, a down quark or a gluon. The right part of Fig. 1 shows the unfolded distribution compared to PYTHIA 6 [7] and HERWIG ++ [8] for the $p_{\mathrm{T}}$ bin $1000<p_{T, 1}<1800 \mathrm{GeV}$. The comparison shows a good agreement between data and simulations.

The differential cross sections of $\mathrm{Z} / \gamma^{*}+$ jets to $\gamma+$ jets and the ratio between the two processes has been studied in [3]. The measurement is performed in various kinematic regions, in this proceeding it is presented the case where $n_{\text {jets }} \geq 1$. Figure 2 shows the ratio (left) of the differential cross sections of $\mathrm{Z}+$ jets over $\gamma+$ jets. The right plot of Fig. 2 shows the comparison of theory (BlackHat [9] and Madgraph [10]+PYTHIA 6) over data. It is shown that the ratio reaches a plateu around $p_{\mathrm{T}} \approx 300 \mathrm{GeV}$ where the mass of $\mathrm{Z}$ becomes less significant in the calculation. Blackhat reproduces data within $10 \%$ while Madgraph overestimates data by $\sim 20 \%$.

The measurement of the dijet azimuthal decorrelations between the two leading jets at $8 \mathrm{TeV}$ has been studied in [5]. This analysis measured the normalized differential cross section as a function of the azimuthal separation of the two leading jets $\left(\Delta \phi_{\text {dijet }}\right)$. The measurement is performed in 

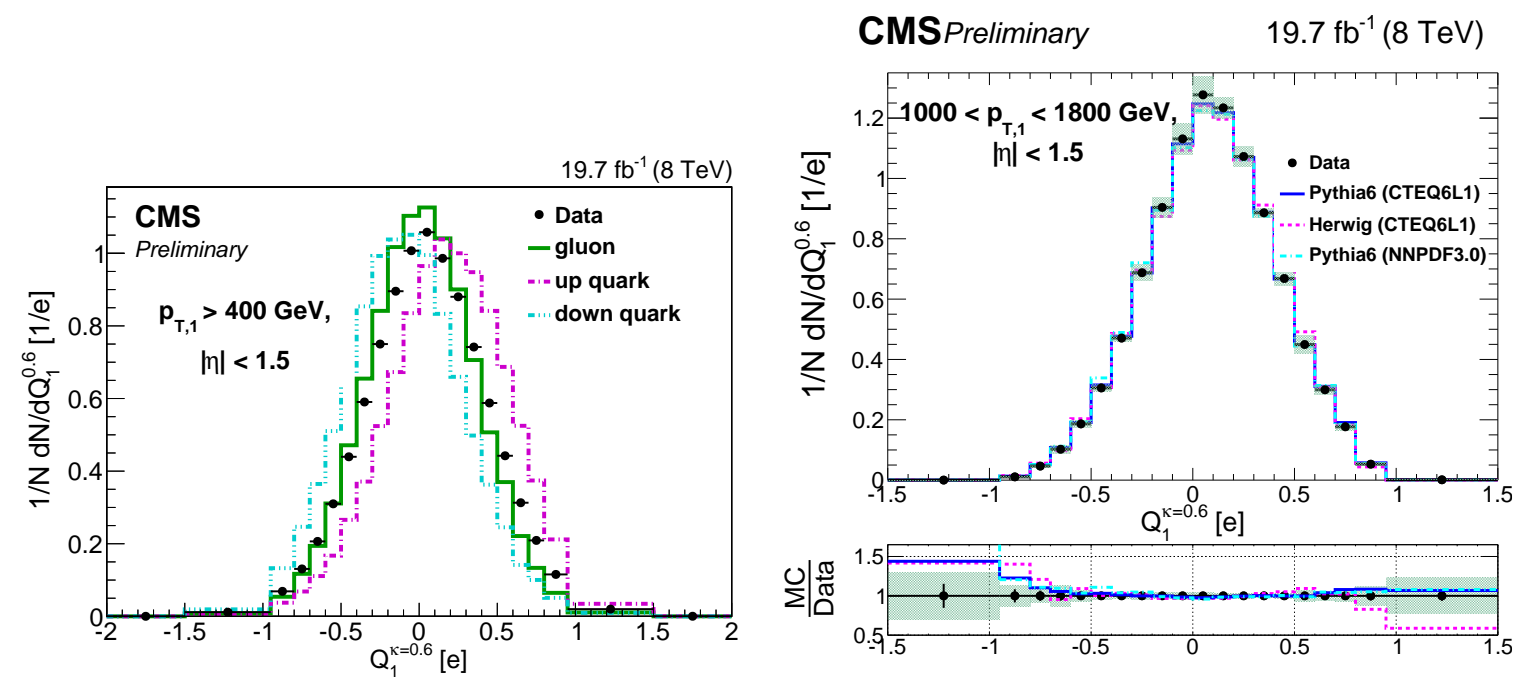

Figure 1: Left: jet charge from data compared to $\mathrm{u}, \mathrm{d}$, and $\mathrm{g}$ distributions derived using PYTHIA 6. Right: unfolded distribution from data compared to predictions from PYTHIA 6 and HERWIG++.
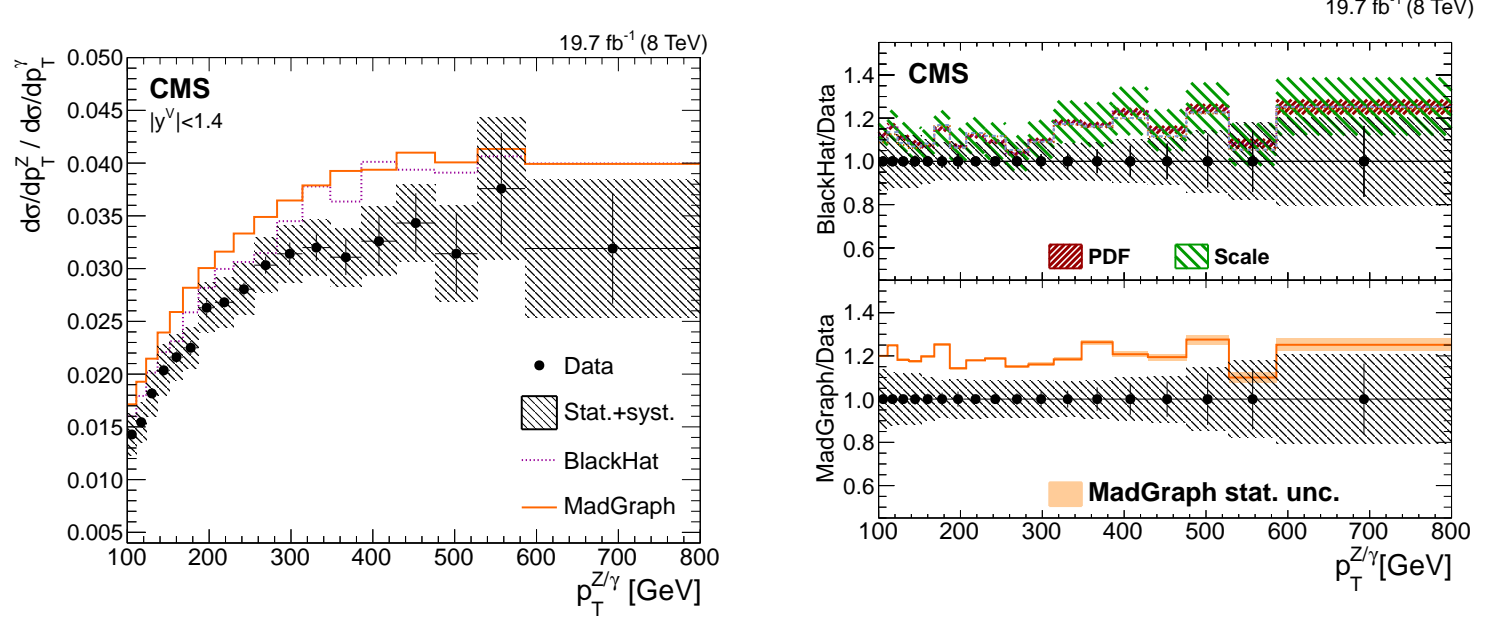

Figure 2: Left: ratio of $\mathrm{Z}+$ jets over $\gamma+$ jets as a function of the total transverse-momentum of the vector bosons. Right: ratio of data to simulations, the error bars represent the statistical uncertainty while the grey band around the data points the total uncertainty in the measurement.

seven bins of the leading jet transverse momentum ( $p_{T}^{\max }$ ) up to $2.2 \mathrm{TeV}$. The dijet azimuthal decorrelation probes effects arising by multijet events by measuring the $\Delta \phi_{\text {dijet }}$ of the two leading jets. Figure 3 shows on the left the data overlaid to theoretical predictions at LO and NLO calculated using NLOJet $++[11,12]$ within the fastNLO [13] framework. The right part of Fig. 3 shows the ratio of various Monte Carlo generators matched to parton showers over data. The comparison between Pythia6 TuneZ2*[14], Herwig++, Pythia8 CUETM1[15, 16], Madgraph5 + Pythia6 TuneZ2*, and POWHEG[17, 18, 19] +Pythia8 CUETM1 shows that Madgraph gives the best description in the 
entire $\Delta \phi_{\text {dijet }}$ region.
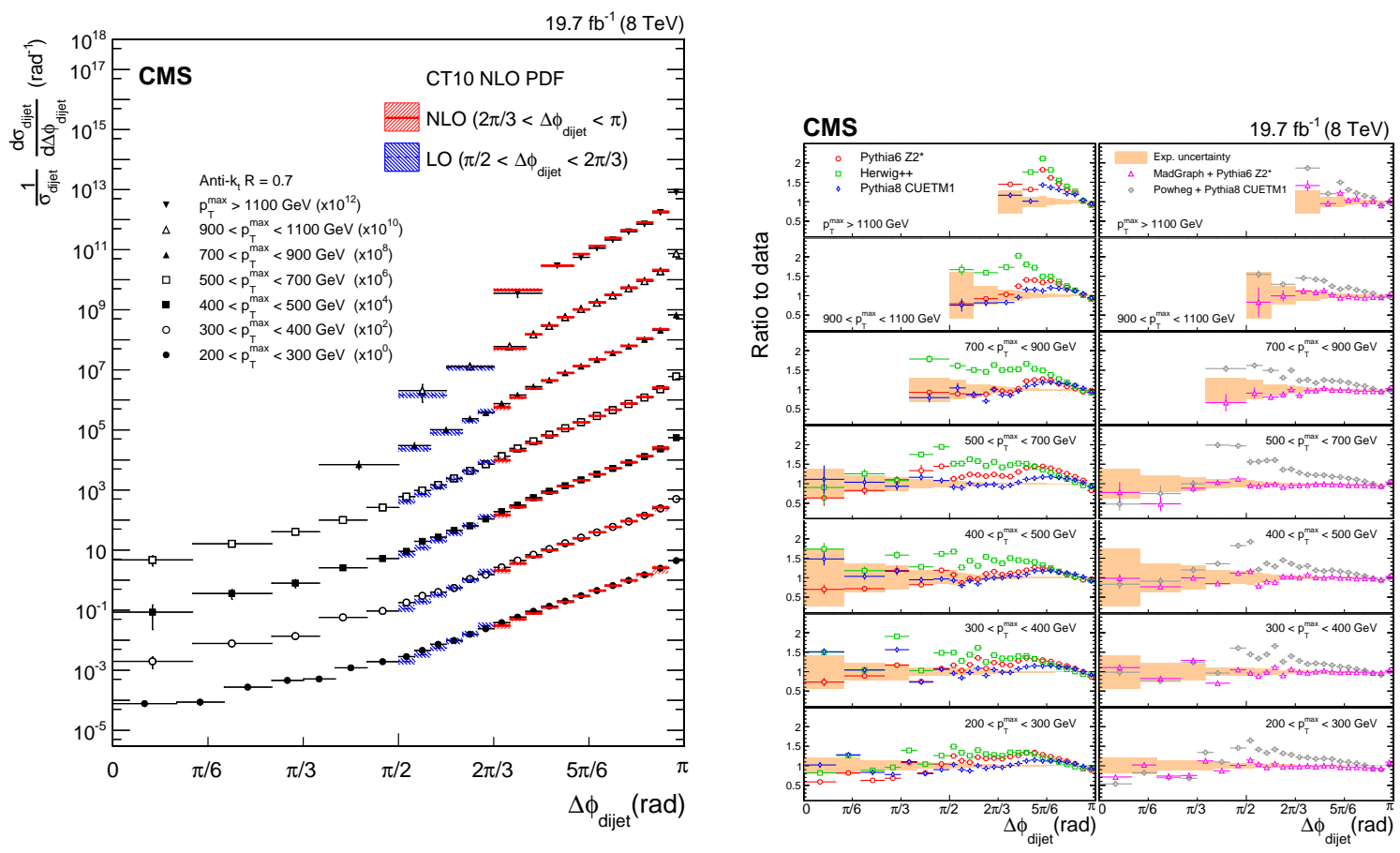

Figure 3: Left: normalised dijet cross section differential in $\Delta \phi_{\text {dijet }}$ for seven $p_{\mathrm{T}}^{\max }$ regions, compared to LO and NLO predictions using CT10 NLO PDF. Right: ratios of data to predictions from various MC generator, for all $p_{\mathrm{T}}^{\max }$ regions.

The measurement of the double differential inclusive jet cross section in $p_{\mathrm{T}}$ and $|y|$ at $8 \mathrm{TeV}$ [4] is reported next. The measurement is performed as a function of $p_{\mathrm{T}}$ in six rapidity bins of $\Delta|y|=0.5$ up to $|y|=3$.0. Jets recorded in a range starting from $74 \mathrm{GeV}$ up to $2.5 \mathrm{TeV}$. The measurement is used to extract the strong coupling and study the PDFs of the proton. Figure 4 shows the ratio of data to theory using various $\alpha_{s}\left(M_{Z}\right)$ values on the left and the impact of the CMS data on the gluon PDF on the right. Theory was calculated using NLOJet ++ with the CT10-NLO PDF set [20], and is corrected for non-perurbative(NP) and electroweak(EWK) effects. It is shown that the inclusion of the CMS data improves the uncertainties of the gluon PDF. Data from all rapidity bins were combined to a $\chi^{2}-$ fit and the strong coupling was found to be:

$$
\alpha_{s}\left(M_{Z}\right)=0.1164_{-0.0015}^{+0.0014}(\exp )_{-0.0029}^{+0.0025}(P D F) \pm 0.0001(N P)_{-0.0028}^{+0.0053}(\text { scale }) .
$$

The running of the strong coupling has also been studied in the same analysis, it is shown in Fig. 5. The asymptotic behaviour of the strong coupling seems to be valid up to $\mathrm{Q} \sim 1.5 \mathrm{TeV}$, which is the maximum reachable from the dataset.

Finally the measurement of the double differential inclusive jet cross section in $p_{\mathrm{T}}$ and $|y|$ at 13 $\mathrm{TeV}[6]$ is reported. The analysis delivers two measurements where jets are reconstructed using the anti- $k_{t}$ algorithm [21] with the distance parameter R, set at 0.4 and 0.7 . The measurement recorded jets with $p_{\mathrm{T}}$ up to $2 \mathrm{TeV}$ and $|y|<4.7$. Left plot of Fig. 6 shows the data, reconstructed with 

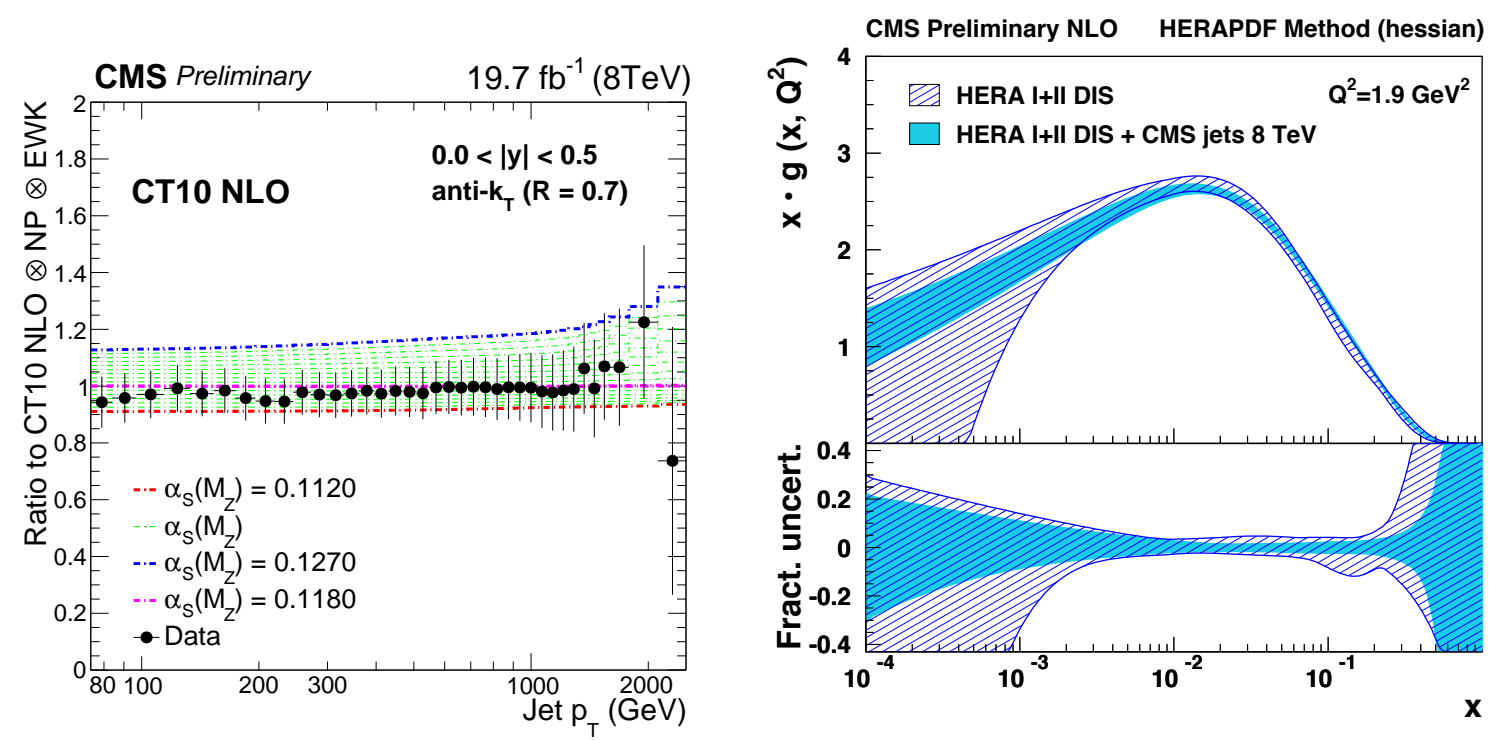

Figure 4: Left: ratio of data over theory predictions for various $\alpha_{s}\left(M_{Z}\right)$ values in the range 0.112-0.127 in steps of 0.001. Right: distribution of gluon as functions of $\mathrm{x}$. The results of the fit to the HERA data and inclusive jet measurements at $8 \mathrm{TeV}$ (shaded band), and to HERA only (hatched band) are compared with their total uncertainties.

$\mathrm{R}=0.7$, compared with predictions from NLOJet ++ , corrected with NP and EWK corrections. Right plot of Fig. 6 shows the data, reconstructed with $\mathrm{R}=0.4$, compared with predictions from POWHEG+PYTHIA8.

Figure 7 shows the ratios of data to NLOJet ++ (top) and the ratio to MC generators matched to parton showers(bottom), using $\mathrm{R}=0.4$ (right) and $\mathrm{R}=0.7$ (left), for the rapidity bin $|y|<0.5$. Calculations using NLOJet ++ are performed with various PDF sets, an overall good agreement is found in the entire range of $p_{\mathrm{T}}$. Calculations with the MC generators show that leading-order generators exhibit significant discrepancies in absolute scale while the NLO generator POWHEG+PYTHIA8 agrees with data. Comparing predictions between NLOJet ++ and POWHEG+PYTHIA8 it is observed that the later gives equally good predictions for both cones while NLOJet ++ overestimates data by $\sim 5-10 \%$ when jets are clustered with $\mathrm{R}=0.4$.

\section{Conclusions}

This proceeding reported five measurements performed by the CMS collaboration with jets and photons in the final state. Comparisons both from fixed-order calculations in pQCD and with $\mathrm{MC}$ generators matched to parton showers were performed. The results from the first $13 \mathrm{TeV}$ data show that the available theoretical tools can describe experiment very well.

\section{Acknowledgements}

This research has been co-financed by the European Union (European Social Fund - ESF) and Greek national funds through the Operational Program "Education and Lifelong Learning" of the 


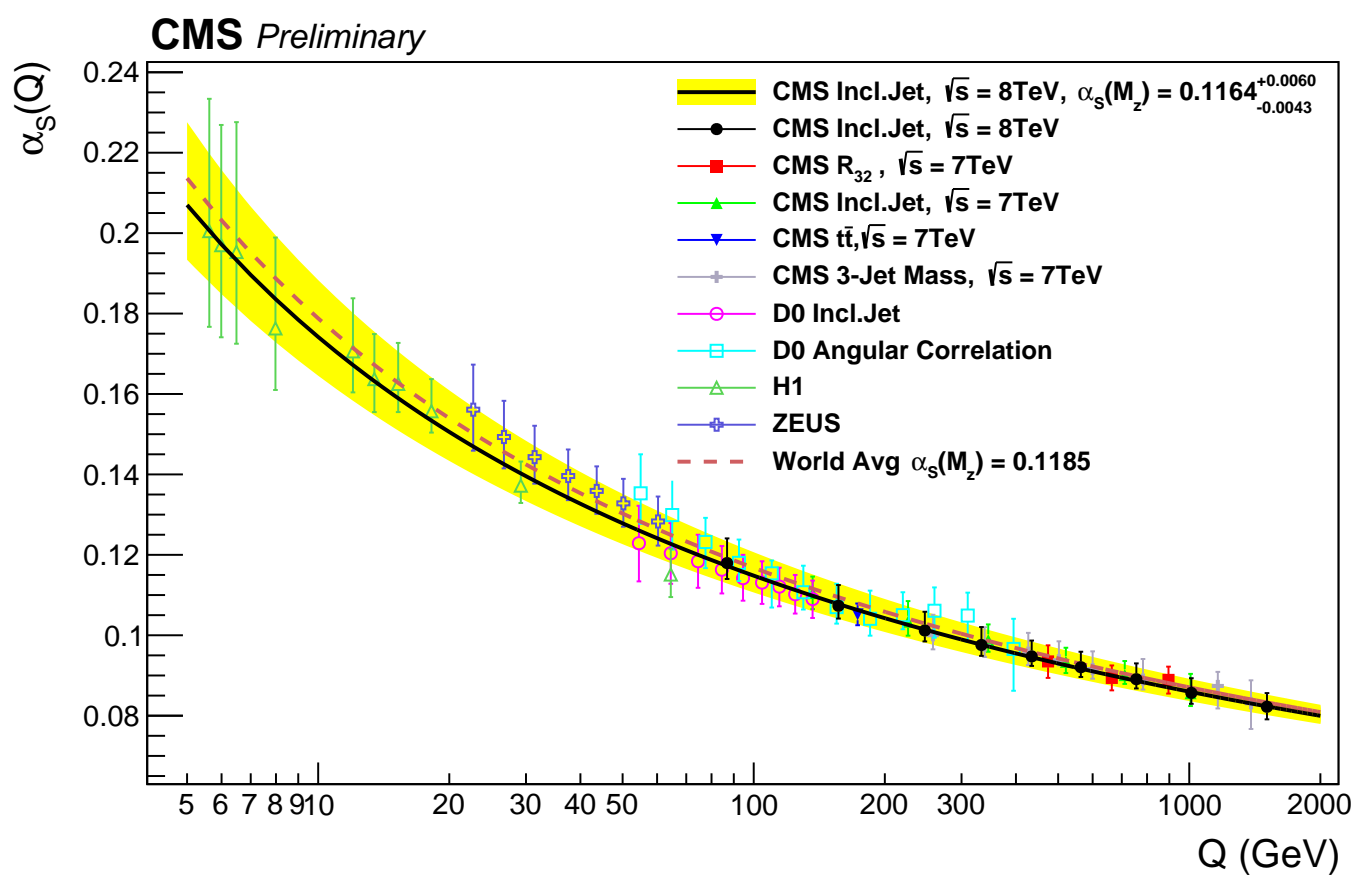

Figure 5: The running $\alpha_{s}(Q)$ as a function of the scale Q is shown, as obtained using CT10 NLO PDF set. The solid line and the uncertainty band are obtained evolving the extracted $\alpha_{s}\left(M_{Z}\right)$ values with the 2-loop 5-flavour RG evolution. The black dots in the figure show the numbers obtained from $8 \mathrm{TeV}$ inclusive jet measurement.

National Strategic Reference Framework (NSRF) - Research Funding Program: ARISTEIA and THALIS. Investing in knowledge society through the European Social Fund. 

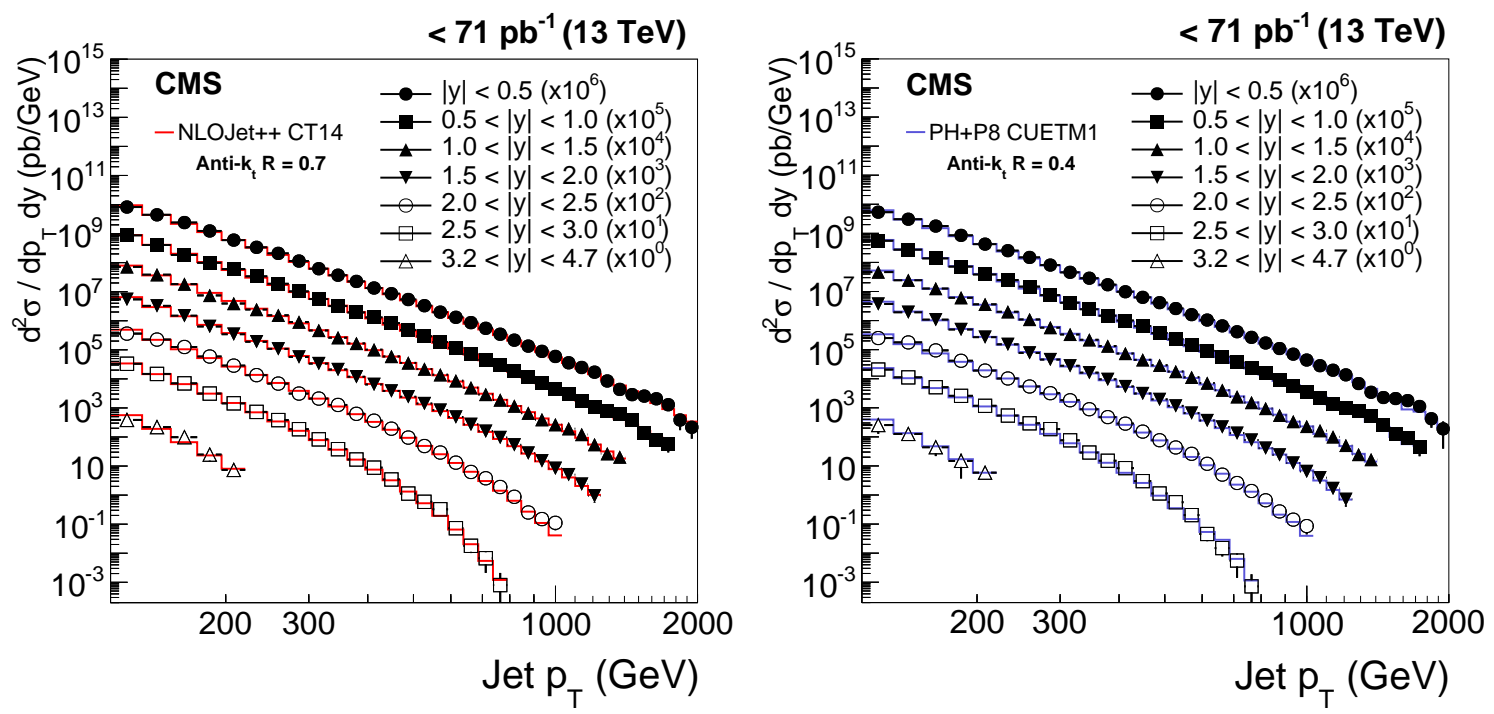

Figure 6: Double-differential inclusive jet cross section as function of jet $p_{\mathrm{T}}$. On the left, data (points) and predictions from NLOJet ++ based on the CT14 PDF set (line) are shown. On the right, data (points) and predictions from POWHEG + PYTHIA-8 (line) are shown.
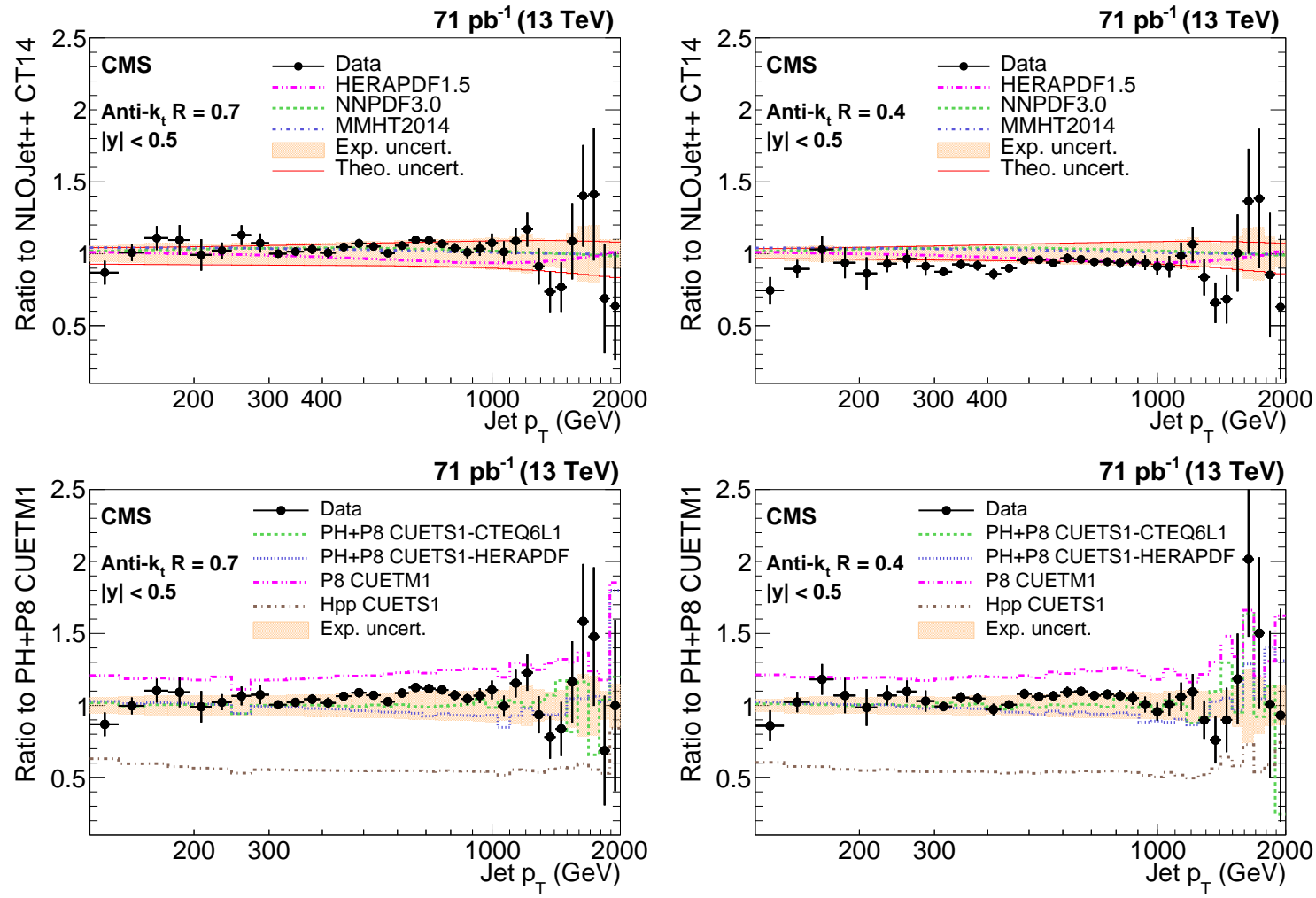

Figure 7: Top: Ratio of measured values to theoretical prediction from NLOJet++ using the CT14 PDF set and corrected for the NP and electroweak effects, using anti- $k_{t}=0.4$ (right) and anti- $k_{t}=0.7$ (left). Bottom: Ratio of measured values to predictions from POWHEG+PYTHIA-8 with tune CUETM1, using anti- $k_{t}=0.4$ (right) and anti- $k_{t}=0.7$ (left). 


\section{References}

[1] CMS Collaboration, The CMS experiment at the CERN LHC, Journal of Instrumentation 3 (08) (2008) S08004.

[2] CMS Collaboration, Measurement of jet charge observables in dijet events at $\sqrt{s}=8 \mathrm{TeV}$ (CMS-PAS-SMP-15-003).

URL https: / / cds. cern. ch/record/2157072

[3] CMS Collaboration, Comparison of the $\mathrm{Z} / \gamma+$ jets to $\gamma+$ jets cross sections in pp collisions at $\sqrt{s}=8$ TeV, JHEP 10 (2015) 128, [Erratum: JHEP04,010(2016)]. arXiv:1505.06520, doi:10.1007/JHEP04(2016)010, 10.1007/JHEP10(2015)128.

[4] CMS Collaboration, Measurement of the double-differential inclusive jet cross section at $\operatorname{sqrt}(\mathrm{s})=8$ TeV (CMS-PAS-SMP-14-001).

URL http: / / cds. cern. ch/record/2060744

[5] CMS Collaboration, Measurement of dijet azimuthal decorrelation in pp collisions at $\sqrt{s}=8 \mathrm{TeV}$, Submitted to: Eur. Phys. J. CarXiv:1602.04384.

[6] CMS Collaboration, Measurement of the double-differential inclusive jet cross section in proton-proton collisions at $\sqrt{s}=13 \mathrm{TeV}$, Eur. Phys. J. C76 (8) (2016) 451. arXiv:1605.04436, doi:10.1140/epjc/s10052-016-4286-3.

[7] T. Sjöstrand, S. Mrenna, P. Z. Skands, PYTHIA 6.4 physics and manual, JHEP 05 (2006) 026. arXiv:hep-ph/0603175, doi:10.1088/1126-6708/2006/05/026.

[8] M. Bähr, S. Gieseke, M. A. Gigg, D. Grellscheid, K. Hamilton, O. Latunde-Dada, S. Plätzer, P. Richardson, M. H. Seymour, A. Sherstnev, B. R. Webber, Herwig++ physics and manual, Eur. Phys. J. C 58 (2008) 639. arXiv:0803.0883, doi:10.1140/epjc/s10052-008-0798-9.

[9] Z. Bern, L. J. Dixon, F. Febres Cordero, S. HÃúche, H. Ita, D. A. Kosower, D. Maitre, Ntuples for NLO Events at Hadron Colliders, Comput. Phys. Commun. 185 (2014) 1443-1460. arXiv:1310.7439, doi:10.1016/j.cpc.2014.01.011.

[10] J. Alwall, M. Herquet, F. Maltoni, O. Mattelaer, T. Stelzer, MadGraph 5: going beyond, JHEP 06 (2011) 128. arXiv:1106.0522, doi:10.1007/JHEP06(2011)128.

[11] Z. Nagy, Three-jet cross sections in hadron-hadron collisions at next-to-leading order, Phys. Rev. Lett. 88 (2002) 122003. arXiv:hep-ph/0110315, doi:10.1103/PhysRevLett.88.122003.

[12] Z. Nagy, Next-to-leading order calculation of three-jet observables in hadron-hadron collisions, Phys. Rev. D 68 (2003) 094002. arXiv:hep-ph/0307268, doi:10.1103/PhysRevD.68.094002.

[13] D. Britzger, K. Rabbertz, F. Stober, M. Wobisch, New features in version 2 of the fastNLO project (2012) 217arXiv:1208.3641, doi:10.3204/DESY-PROC-2012-02/165.

[14] CMS Collaboration, Study of the underlying event at forward rapidity in pp collisions at $\sqrt{s}=0.9,2.76$, and 7tev, JHEP 2013. arXiv:1302.2394, doi:10.1007/JHEP04(2013)072.

[15] T. Sjöstrand, S. Mrenna, P. Z. Skands, A Brief Introduction to PYTHIA 8.1, Comput. Phys. Commun. 178 (2008) 852. arXiv:0710.3820, doi:10.1016/j.cpc.2008.01.036.

[16] CMS Collaboration, Event generator tunes obtained from underlying event and multiparton scattering measurements, Eur. Phys. J. C76 (3) (2016) 155. arXiv:1512.00815, doi:10.1140/epjc/s10052-016-3988-x. 
[17] S. Frixione, P. Nason, C. Oleari, Matching NLO QCD computations with parton shower simulations: the POWHEG method, JHEP 11 (2007) 070. arXiv:0709.2092, doi:10.1088/1126-6708/2007/11/070.

[18] S. Alioli, P. Nason, C. Oleari, E. Re, A general framework for implementing NLO calculations in shower monte carlo programs: the POWHEG BOX, JHEP 06 (2010) 043. arXiv:1002.2581, doi:10.1007/JHEP06(2010)043.

[19] P. Nason, A new method for combining NLO QCD with shower Monte Carlo algorithms, JHEP 0411 (2004) 040. arXiv:hep-ph/0409146, doi:10.1088/1126-6708/2004/11/040.

[20] H.-L. Lai, M. Guzzi, J. Huston, Z. Li, P. M. Nadolsky, et al., New parton distributions for collider physics, Phys. Rev. D 82 (2010) 074024. arXiv:1007.2241, doi:10.1103/PhysRevD.82.074024.

[21] M. Cacciari, G. P. Salam, G. Soyez, The Anti-k(t) jet clustering algorithm, JHEP 04 (2008) 063. arXiv:0802.1189, doi:10.1088/1126-6708/2008/04/063. 